\title{
LA DECOLONIALIDAD DEL PATRIMONIO: LAS FUENTES DE SABERES Y SISTEMAS DE SABERES EN LA POBLACIÓN HETEROGÉNEA DE COSTA RICA
}

Recibido: 28-08-2016

Aprobado: 24-10-2016

\author{
Kirsten Grünberg \\ Yonkers Riverfront Library, Nueva York
}

Kirsten Grünberg.

Posee una Maestría en

Educación de Framingham State University y otra Maestría en las Ciencias de la Información con énfasis en Humanidades Digitales de Pratt Institute. Además, realizó estudios en el Doctorado en Estudios de la Sociedad y la Cultura de la Universidad de Costa Rica.

kike.grunberg@gmail.com

\begin{abstract}
RESUMEN
El eje fundamental del documento constituye en explorar el concepto de "el patrimonio" dentro de la estructuras propias de "la colonialidad", específicamente aquellas que han permeado en la población costarricense debido a las políticas culturales llevadas a cabo por las instituciones. La propuesta también desea sugerir una serie de alternativas para sustituir el concepto de "el patrimonio" por medio de posturas propias de "la decolonialidad". En este sentido, se desea enfatizar la fuentes de saberes y sistemas de saberes como medios que fortalezcan las diversas identidades de la población heterogénea de Costa Rica.
\end{abstract}

Palabras clave: patrimonio, decolonialidad, saberes, indígena, Costa Rica.

\begin{abstract}
The purpose of the document is to explore the concept of "patrimony" within the dominant structure of "colonialism", as a result of the institutional cultural policies implemented throughout the Costa Rican population. The document also suggests a series of alternatives to substitue the concept of "patrimony" by applying a series of "decolonial" approaches. Within this context, knowledge sources and knowledge organizations are emphasized as a means to affirm the multiple identities of the heterogenous population of Costa Rica.
\end{abstract}

Keywords: patrimony, decolonial, knowledge, indigenous, Costa Rica.

\section{INTRODUCCIÓN}

El eje fundamental del documento constituye en explorar el concepto de "el patrimonio" dentro de la estructura dominante de "la colonialidad" que ha permeado en la población costarricense, debido a las políticas culturales establecidas a nivel institucional. La 
"colonialidad" se refiere a las estructuras de poder y conocimientos eurocéntricos que se instalan en América a partir de 1492 (Wikipedia, 2016a), mientras que sus procesos de legitimación institucional revelan "las tramas de la interdiscursividad/interseccionalidad, donde es posible relevar lo que el discurso del poder patriarcal oculta o pervierte" (Palermo, 2012, p.17). Por tanto, a partir del discurso de "el patrimonio", se seleccionan un conjunto de expresiones, se tergiversan las cosmovisiones de las cuales se desprenden y se invisibilizan las diversas dinámicas sociales de donde provienen.

La propuesta también desea sugerir una serie de alternativas para sustituir el concepto de "el patrimonio" por medio de posturas propias de "la decolonialidad", entendida aquí como procesos que promueven y generan alternativas constructivas ante las estructuras de la "colonialidad" (Restrepo \& Cabrera, s.f.). En este sentido, se desea enfatizar la fuentes de saberes y sistemas de saberes como medios que fortalezcan las diversas identidades de la población heterogénea de Costa Rica.

También es importante aclarar que el presente análisis no logrará liberarse o purgarse por completo de las características propias de "la colonialidad", así como ofrecer propuestas completamente elaboradas dentro de parámetros "decoloniales", ya que se recurre al mismo idioma español y a fuentes académicas para redactar este texto. Consciente de esta realidad, se invita a los diferentes sectores de la población para continuar el diálogo del presente tema.

\section{EXPLORACIÓN DEL CONCEPTO DE "EL PATRIMONIO" Y SU DIFUSIÓN INSTITUCIONAL}

La palabra "patrimonio" significa "lo recibido por línea paterna" y parte de las raíces latinas patri ('padre') y monium ('recibido') (Wikipedia, 2016b). El concepto fue desarrollado a partir del derecho romano el cual establecía que la propiedad se heredaba y se transmitía de generación a generación. Por su parte, Mariano Marcos Andrade Butzonitch $(2009$, p.11) afirma que la figura paterna también es heredada a través de la patria y expresa la siguiente reflexión al respecto:

Culturalmente, el significante pater, puede ser todo menos inocuo en la historia de la civilización occidental y cristiana a la que debemos, entre otras cosas, estas instituciones. En el caso del patrimonio cultural, aquél significante transporta el símbolo de un poder inscrito en la sangre a través 
de la herencia y trasladado al campo de la ley y el Estado, como modelo cívico y prototipo identitario de la Nación.

Por tanto, el concepto "patrimonio" y sus derivaciones - patrimonio tangible, patrimonio intangible, patrimonio cultural, patrimonio natural, etc. - son producto de iniciativas hegemónicas del Estado o los Estados en su integración internacional que, de acuerdo con Butzonitch (2009, p.25), responden a "estrategias capitalistas para ubicar a la cultura en un lugar funcional para sus intereses".

Una definición del concepto de "patrimonio" que se aplica en diversas instituciones de Costa Rica, fue desarrollada por el Instituto Latinoamericano de Museos y Parques (Fundación ILAM). Esta definición mantiene una perspectiva funcional y es entendida como:

el conjunto de bienes culturales y naturales, tangibles e intangibles, generados localmente, y que una generación hereda y transmite a la siguiente con el propósito de preservar, continuar y acrecentar dicha herencia (DeCarli, 2006, en Fundación ILAM, 2016).

Por su parte, el Ministerio de Cultura y Juventud brinda un contexto descriptivo de un conjunto de bienes, ya que afirma que el patrimonio cultural material comprende

los objetos o bienes materiales que reflejan cómo los costarricenses nos hemos adaptado al medio y organizado nuestra vida social, económica y cultural. Ejemplos de ello son las obras de arquitectura, obras de arte, cerámica, orfebrería, muebles, documentos, piezas arqueológicas, instrumentos musicales, herramientas (Ministerio de Cultura y Juventud de Costa Rica, s.f.a).

Además, el Ministerio de Cultura y Juventud desarrolla el concepto patrimonio cultural inmaterial a partir de la definición de la Organización de las Naciones Unidas para la Educación, la Ciencia y la Cultura (UNESCO), el cual se define como

\footnotetext{
las tradiciones orales, artes del espectáculo, usos sociales, rituales, actos festivos, conocimientos y prácticas relativos a la naturaleza y el universo, y saberes y técnicas vinculados a la artesanía tradicional (Ministerio de Cultura y Juventud de Costa Rica, s.f.b).
}

Sin embargo, la Política General de Derechos Culturales 2014-2023 del Ministerio de Cultura y Juventud (2013) desgloza el concepto de "patrimonio" y sus aplicaciones con mayor profundidad. 
Desde el punto de vista de "la colonialidad", los conceptos patrimoniales hacen caso omiso a dos aspectos fundamentales: la cuestión social y las fuentes de saberes/sistemas de saberes de la población heterogénea de Costa Rica. En este sentido, las propuestas de patrimonio del ILAM y el Ministerio de Cultura excluyen "las contradicciones sociales y los antagonismos de clase" (Briceño Porras, 2011 , p.3) o, en otras palabras, se encuentra ausente la cuestión social que refleja las desigualdades y antagonismos delimitados en un campo geográfico y temporal (lanni y Rose en De Sena, 2014, p.154). Por tanto, la ausencia de las diversas dinámicas sociales en ambas propuestas de patrimonio invisibiliza los vínculos afectivos y las pugnas sociales de los gestores de los bienes tangibles e intangibles, y más peligroso aún, oculta a aquel conjunto de personas que también las va a transmitir y a recibir.

A su vez, las propuestas de patrimonio del ILAM y el Ministerio de Cultura aplican el conocimiento occidental para definir las múltiples expresiones tangibles e intangibles, una visión que limita las demás fuentes de saberes/sistemas de saberes presentes en la población heterogénea de Costa Rica. Estas prácticas del conocimiento occidental son analizadas desde "la colonialidad" de la siguiente manera: Henrich (2014, p.43) afirma que la cientificidad y la universalidad del conocimiento es difundido e impuesto en diferentes ámbitos institucionales mediante diversos dispositivos de poder, y funciona como un modelo civilizatorio que refleja "la colonialidad del saber"; mientras que Walsh (2004) explica que "se mantiene la hegemonía del eurocentrismo como única perspectiva de conocimiento" y surgen sistemas de control caracterizados por una serie de lenguajes y clasificaciones jerárquicas. Un ejemplo de "la colonialidad del saber" es que ambas propuestas de patrimonio ILAM y el Ministerio de Cultura - consagran la memoria como una "forma particular de traer el pasado hasta el presente, propia de aquellas sociedades con una noción lineal del tiempo" (Molina, 2007, p.130).

Las diversas instituciones culturales y académicas adaptan los conceptos patrimoniales de acuerdo a sus intereses y necesidades. Este conjunto de prácticas también se encuentra determinado por la influencia de las élites y no por las diferencias sociales que concurren en el seno de la sociedad nacional, tal y como lo expresa Cecilia Benedetti en Antropología social y patrimonio; perspectivas teóricas 
latinoamericanas (en Briceño Porras, 2011, p.248). Por tanto, el gobierno costarricense y las diversas instituciones del país han creado sus colecciones patrimoniales a partir de los objetos ceremoniales, funerarios y restos humanos de las comunidades indígenas y, además, han tomado ventaja para crear muchos de sus parques nacionales o reservas biológicas en tierras indígenas o aledaños a ellas. Desde una perspectiva occidental, la soberbia, la arrogancia, la alcagüetería, el desconocimiento y la falta de interés por conocer y respetar el derecho de los miembros de las comunidades indígenas nos ha hecho cómplices - y, a la vez, responsables - de no respetar sus territorios ancestrales, su derecho colectivo a su integridad y, más grave aún, el derecho a su supervivencia colectiva. El solo hecho de no respetar su territorio ancestral "les ocasionan sufrimiento, y perjudican la preservación de su forma de vida, costumbres e idioma." (MacKay \& Morales Garro, 2014, p.36).

Las instituciones culturales

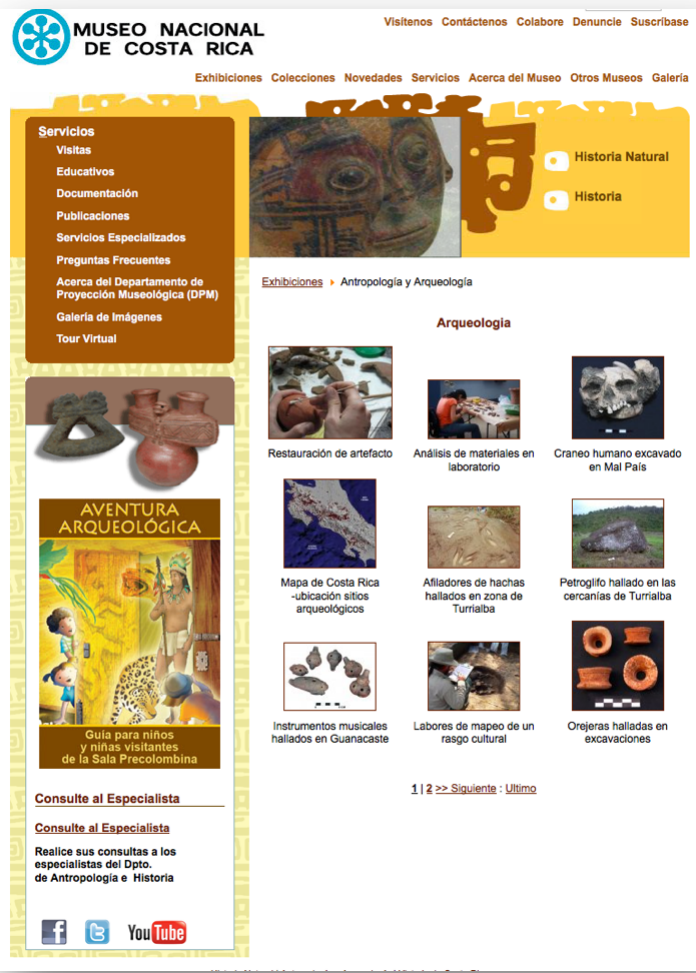
tampoco se han tomado la molestia de realizar una consulta previa a las comunidades indígenas para incorporar las imágenes digitales en sus medios de difusión, los cuales corresponden a sus objetos ceremoniales, funerarios y restos humanos (Grünberg, 2015), tal y como lo muestra la página del Museo Nacional de Costa Rica.

Imagen de la página de internet. Arqueología. Museo Nacional de Costa Rica. ${ }^{1}$

Otro aspecto lamentablemente, es la reciente publicación del Manual de

${ }^{1}$ Dirección:

http://www.museocostarica.go.cr/index.php?option=com_zoom\&ltemid=63\&catid=7\&lang= es_CR.

Consultado el 9 de Agosto de 2016. 
normas y procedimientos para el tratamiento y el manejo de las colecciones arqueológicas con contexto del Museo Nacional de Costa Rica, ya que la publicación hace caso omiso al conjunto de tratados internacionales ligados a los pueblos indígenas. Por ejemplo, el Museo Nacional de Costa Rica no solo tiene la audacia de asumir la autoridad de otorgar el "valor" que se merecen las naciones y las comunidades indígenas sino que, además, construirán, sobre este fundamento, a la sociedad costarricense (Museo Nacional de Costa Rica, 2016, p.8). Sin embargo, también es importante destacar que las naciones y comunidades indígenas del mundo han ganado mucho terreno en el campo museístico, el cual se ve reflejado en el siguiente apartado:

Cuando en las colecciones museísticas esté representado el patrimonio cultural de pueblos indígenas, los Estados Miembros deberían adoptar las medidas apropiadas para alentar y facilitar el diálogo y la creación de relaciones constructivas entre esos museos y los pueblos indígenas respecto de la gestión de esas colecciones y, si procede, hacer devoluciones o restituciones a tenor de las leyes y políticas aplicables (Organización de las Naciones Unidas para la Educación, la Ciencia y la Cultura, 2015).

La rectificación del artículo de la UNESCO pretende corregir las ideologías y las prácticas coloniales llevadas a cabo por los museos, las bibliotecas, los archivos y otras instituciones culturales que, a lo largo de su historia institucional y bajo la mirarada científica, se han apropiado indebidamente de restos humanos indígenas y sus diversas expresiones, interpretadas indebidamente como patrimonio cultural. Por tanto, es indispensable estar pendientes de la legislación nacional, así como los tratados internacionales de El Convenio 169 de la Organización Internacional del Trabajo sobre pueblos indígenas y tribales ${ }^{2}$ y la Declaración de las Naciones Unidas sobre los Derechos de los Pueblos Indígenas ${ }^{3}$ que, de acuerdo a la Constitución Política de Costa Rica, mantienen una autoridad superior a las leyes

\footnotetext{
${ }^{2}$ International Labor Organization. (2009). Indigenous and Tribal Peoples' Rights in Practice. A Guide to the ILO Convention No.169. Recuperado de http://www.ilo.org/wcmsp5/groups/public/---dgreports/---dcomm/--publ/documents/publication/wcms_171810.pdf

${ }^{3}$ Naciones Unidas. (Marzo 2008). Declaración de las Naciones Unidas sobre los derechos de los pueblos indígenas. Recuperado de http://www.un.org/esa/socdev/unpfii/documents/DRIPS_es.pdf
} 
costarricenses ${ }^{4}$. Es fundamental que en Costa Rica se tomen las medidas necesarias para desmantelar el concepto de patrimonio institucional vigente con el fin de respetar el desarrollo de las distintas visiones y múltiples voces de las comunidades indígenas.

Desde un contexto de "la colonialidad", los campos de legitimaciones patrimoniales en Costa Rica también parten de otra serie de rasgos de inclusión y exclusión en la población heterogénea del país. Estas prácticas con frecuencia sugieren la producción de "un catálogo preciso del ser costarricense" donde se afirma el conocimiento sobre los límites, las falsedades y los gestos de una comunidad cuya representación sigue en pugna (Baltodano en Román \& Camacho Alfaro, 2010, p.13). Por ejemplo, después de estudios académicos sumamente serios y exhaustivos, "La tradición del boyero y las carretas de Costa Rica" fueron declaradas Obra Maestra del Patrimonio Oral e Inmaterial de la Humanidad por la UNESCO el 24 de noviembre del 2005 (Organización de las Naciones Unidas para la Educación, la Ciencia y la Cultura, 2006) (Wikipedia, 2016c) (Organización de las Naciones Unidas para la Educación, la Ciencia y la Cultura, 2005) y su décimo aniversario fue organizado por la Asociación Boyera Costarricense y el Ministerio de Cultura y Juventud (La Prensa Libre, 2015). Sin embargo, a pesar de que la declaración patrimonial haya sido aceptada oficialmente por algunos sectores institucionales, nacionales e internacionales, existen otros representantes de la sociedad costarricense que le han otorgado connotaciones distintas a "la carreta típica" o al símbolo patrio del "trabajo". En este sentido, Carlos Cortés (en Baltodano Román \& Camacho Alfaro, 2010, p.195) expresa que actualmente la carreta típica "ha sido sublimimado hasta convertirse en un souvenir decorativo" y expresa el siguiente argumento al respecto:

Si alguno de usteds ha visto la "carreta típica" verá que ha "blanqueado" de la misma manera que la ideología del mestizo blanco ha hecho con la sociedad costarricense - su primitivo carácter utilitario por el suntuario. Es decir, le ha "borrado la tierra" el capital, la estructura productiva, etc. -, los

\footnotetext{
${ }^{4}$ Artículo 7: Los tratados públicos, los convenios internacionales y los concordatos debidamente aprobados por la Asamblea Legislativa, tendrán desde su promulgación o desde el día que ellos designen, autoridad superior a las leyes. La Nación. (s.f.). Constitución Política de Costa Rica. Recuperado de http://wvw.nacion.com/In_ee/ESPECIALES/constitucion/docu1.html
} 
trazos, los vestigios de la producción, y ha privilegiado la decoración - la superestructura ideológica, como se decía antes.

Dentro del mismo contexto, se puede referir al Museo de la Carreta y El Campesino Costarricense, el cual fue declarado de interés histórico y patrimonial en 1982. La Municipalidad de Desamparados manifiesta que, aunque la declaratoria de "La tradición del boyero y las carretas de Costa Rica" haya despertado interés, en la actualidad el museo "se encuentra en un estado deplorable" (Municipalidad de Desamparados, s.f.).

Por tanto, aunque la selección de algunos bienes tangibles e intangibles hayan sido "amparados" por las legitimaciones patrimoniales, ello no garantiza que éstos sean legitimados por diversos sectores del país o que se encuentren cubiertos en su totalidad por acciones que faciliten y garanticen "preservar, continuar y acrecentar dicha herencia" (DeCarli, 2006, en Fundación ILAM, 2016) dentro de un contexto social coherente o de co-herencia.

\section{LA POBLACIÓN DE COSTA RICA Y SUS FUENTES DE SABERES/SISTEMAS DE SABERES}

Como respuesta a los procesos de "la deconolianidad" del patrimonio, se ofrece la propuesta de la visibilidad de las fuentes de saberes/sistemas de saberes de los diferentes sectores sociales de la población heterogénea de Costa Rica. Por este motivo es insdispensable incorporar tanto la representación de todas las poblaciones, como las dinámicas sociales que se desarrollan en el seno de sus comunidades, tal y como lo expresa Butzonitch (2009, p.38):

\footnotetext{
Sólo reconociendo este espacio abierto, indeterminado, para la construcción de cultura desde lo social, donde pueda expresarse el otro, donde se dé cabida y expresión a la diversidad y la diferencia, donde se fomenten intervenciones que permitan la expresión de los sectores marginados y excluidos en el campo económico y político, será posible restablecer el proceso del hacer social que suscite una verdadera democracia. Un modo de hacer política que propicie la construcción de autonomía y el desarrollo de diferentes mundos de la vida, amenazados hoy en día por la primacía de una cultura capturada por la lógica de la mercancía y la globalización indiscriminada de factores economicistas, restrictivos y excluyentes.
} 
Los diferentes sectores sociales de la población heterogénea de Costa Rica pueden entenderse también como "sistemas autopoiéticos", o sea, aquellos sistemas que tienen una organización que les define una identidad propia (autos) y tienen una estructura que es capaz de producirse (poien), es decir, "sistemas que se producen a sí mismos continuamente" (Mejía, 2008). Por ejemplo, algunos grupos sociales en Costa Rica pueden distinguirse por su origen (indígena y extenso mestizaje entre indígenas, europeos, asiáticos y africanos), su condición legal en el país (nacimiento, nacionalización, turista, pensionado o refugiado), o por algunos otros indicadores estadísticos, tal y como lo demuestra el Estado de la Nación (Estado de la Nación, 2015, p.76).

Con respecto al conocimiento, los diferentes sectores sociales de la población heterogénea de Costa Rica construyen los saberes los cuales se mantienen dentro de sistemas organizados. Con el fin de visibilizar y revitalizar este conjunto de saberes, es necesario aplicar diversos procesos de "descolonización" en el campo del saber, entendidos como aquellos que propicien la construcción de la autonomía y el desarrollo de diferentes mundos de la vida (Butzonitch, 2009, p.38). Amawtay Wasi (en Walsh, 2004) propone los siguientes pasos para estos procesos:

- El camino hacia la descolonización requiere hacer ver que el conocimiento tiene valor, color, género y lugar de origen y, por eso, el lugar desde uno piensa sí importa.

- El camino hacia la descolonización requiere la recuperación, revaloración y aplicación de los saberes ancestrales, pero también requiere un cuestionamiento de temporalidad y localidad asociado con ellos, que siempre los mantendrán como "saberes" y no "conocimiento".

- El camino hacia la descolonización no debe partir simplemente de un relacionar de conocimientos (ancestrales, occidentales, orientales) entendidos como bloques o entidades claramente identificados y encerrados, sino en contribuciones críticas a nuevos procesos de intervención intelectual, en la creación de conocimientos y de modos de pensar que cruzan fronteras.

Como respuesta a procesos de "la descolonización", Sousa Santos (en Henrich, 2014, p.45) también propone el concepto de la "ecología de saberes" el cual demanda igualdad en la relación entre las diferentes formas de conocimientos. En este sentido, el autor señala que "en muchas áreas de la vida la ciencia moderna se ha mostrado una superioridad incuestionable con relación a otras formas de conocimiento. Pero también hay otras intervenciones en el mundo 
real que son valiosas y en las que la ciencia no ha tomado parte, como la preservación de la naturaleza por parte formas de conocimientos rurales y campesino-indígenas".

Dentro del ámbito de los saberes y sus sistemas de organización, también se debe de tomar en cuenta un "sistema lingüístico heterogéneo" (Ming, s.f.), o sea,

la variación lingüística como el conjunto de diferentes formas alternativas para expresar un mismo significado en el dominio de una lengua. Es decir, distintos hablantes, o incluso el mismo hablante en distintos momentos, usan formas diferentes para expresar el mismo concepto, o tienen distintas pronunciaciones para la misma palabra. (Wikipedia en Ming, s.f.)

De esta forma, también se logra visibilizar el lenguaje al cual recurre diferentes sectores de la población y tomar en cuenta sus variables, tales como el género, la edad, y papel que juega dentro de la comunidad.

\section{LOS INDÍGENAS Y SUS FUENTES DE SABERES/SISTEMAS DE SABERES}

De acuerdo con el documento Violaciones de los derechos territoriales de los pueblos indígenas: el ejemplo de Costa Rica (MacKay \& Morales, 2014), existen ocho pueblos indígenas en Costa Rica con una población de 104,143 personas, representando aproximadamente el 2.4 por ciento de la población nacional.

Muchos de ellos viven en 24 territorios indígenas titulados y reconocidos legalmente así como en tierras ocupadas tradicionalmente pero que actualmente no se encuentran incluidas dentro de estos territorios con título. (...) Al contrario de la mayoría de los demás estados americanos, no existen garantías constitucionales para los derechos a la propiedad ni los derechos culturales indígenas, con la única excepción siendo el reconocimiento constitucional de los derechos lingüísticos indígenas en 1999.

Con respecto a las fuentes de saberes/sistemas de saberes de los indígenas, éstos parten de las cosmovisiones de cada una de sus comunidades, y una serie de principios las mantienen ordenadas y en equilibrio (Fondo Indígena, 2007, p.53). Por ejemplo, uno de estos principios es el de la relacionalidad:

Este principio es el más importante para nosotros, sin él no podrían existir los demás. Este principio nos dice que todo está vinculado con todo, lo cual nos lleva a afirmar que lo más importante para nosotros no son necesariamente los seres en sí mismos 
sino las relaciones, los vínculos que se establecen entre ellos... Estos vínculos son de varios tipos, pueden ser afectivos, ecológicos, éticos, estéticos o productivos. Todos a la vez o intercalándose. Ni siquiera lo divino, lo sagrado, está fuera de este principio... (Fondo Indígena, 2007, p.55-56)

Por ejemplo, de acuerdo con María Eugenia Bózolli (en García Segura, 2003, p.xiii), los indígenas Bribri mantienen una relación entre el tiempo y lugar, el cual se ve reflejado en una misma palabra ká (o kó según el dialecto) (García Segura, 2003, p.xv-xvi). Otro ejemplo es la relación que mantienen entre el nacimiento y la muerte, o sea, entre ditsö 'semilla', dicha 'huesos' y sula 'origen of principio':

\footnotetext{
Estos tres conceptos tienen que ver con origen e identidad; significan identidad o continuidad, siempre y cuando se mantengan en la condición de semilla viable, es decir cuidada, preservada, guardada. Representan la union de arriba y abajo. (...) De esta unión se derivan las equivalencies entre el nacimiento y la muerte. Este ultimo concepto es el pasaje o regreso al lugar de origen /sulàkasaka/. Las equivalencies entre el nacimiento y la muerte se derivan de la utilización de símbolos relativos a la continuidad societaria, a la inmortalidad social (García Segura, 2003, p.xiii).
}

Por tanto, todas las relaciones se encuentran entretejidas y vivas en las cosmovisiones de las diversas comunidades indígenas en Costa Rica y no requieren ninguna estructrua ajena a ella para su activación.

La propuesta para respetar las cosmovisiones de las diversas comunidades indígenas del país parte tanto de la legislación nacional y de los tratados internacionales, como del conjunto de intereses, necesidades y recomendaciones específicas que generan las mismas comunidades indígenas. También es sumamente importante respetar la postura de no compartir los saberes con personas ajenas a sus comunidades. Estas referencias se vuelven indispensables para responder adecuadamente cualquier gestión que se desea llevar a cabo con una comunidad indígena. Por ejemplo, con el fin de llevar a cabo una consulta previa con las comunidades indígenas, se puede recurrir a la fuente ofrecida en la Consulta y Participación de los Pueblos Indígenas (Instituto Interamericano de Derechos Humanos, 2016, p.137-138). Además, el Perfil de los pueblos indígenas de Costa Rica fue realizado por representantes indígenas con el fin de "incrementar y facilitar el acceso a información sobre asuntos de desarrollo indígena a todas las partes interesadas, incluyendo organizaciones y comunidades indígenas, organizaciones no gubernamentales (ONG) e instituciones académicas" (Guevara Berger, 2007). 


\section{LA POBLACIÓN HETEROGÉNEA Y SUS FUENTES DE SABERES/SISTEMAS DE SABERES}

Además de los indígenas, el documento Afrodescendientes y Multiculturalismo según el Censo 2011 (Campbell Barr, s.f., p.3) describe a la sociedad multicultural como aquella que incluye poblaciones afrodescentientes - negro(a) y mulato(a) y chinas, mientras que no separa las categorías blanco(a) y mestizo(a), colocándola como una sola opción. Cada una de estas poblaciones y sus múltiples expresiones merecen su debida atención y análisis exhaustivo. Sin embargo, tanto la cantidad de sectores sociales como su conocimiento y sistemas de organización no se podrán aportar de manera comprensiva en este documento por motivos de espacio.

A modo de ejemplo, el conocimiento y los sistemas de organización de la población hetereogénea de Costa Rica han estado expuestos, en menor o mayor grado, a esquemas de representación de origen occidental. De acuerdo con Jaén García (2006, p.6), el conocimiento se puede clasificar de la siguiente manera en el campo de la archivística: los reales, que se clasifican como naturales (el ser humano no ha intervenido) o artificiales (el ser humano sí ha intervenido); y los lógicos son los que no desempeñan ninguna actividad. En nombre de la ciencia, este tipo de sistema de clasificación ha sido producto de los avances científicos, lo cuales han tendido a desmantelar los sistemas de conocimiento propios de las poblaciones en Costa Rica.

Sin embargo, con el fin de explorar algunas posibilidades dentro de la población heterogénea, se puede referir al siguiente concepto que fue planteado por el autor costarricense Alberto Cañas (2010): "El chunche, en Costa Rica, lo es todo; es la palabra por excelencia; la que nos sirve a los costarricenses para designar todas las cosas: tanto aquellas cuyo nombre ignoramos, como aquellas de cuyo nombre no quisiéramos acordarnos" (p. 131) . El autor también afirma que: "Con cada nueva palabra que el diccionario acepta, acepta al mismo tiempo la palabra chunche, que es sinónimo de todas." (Cañas, p. 137). De igual forma, al "chunche" se le pueden añadir calificativos 
como corrongo ${ }^{5}$, chuzo $^{6}$ o chiva ${ }^{7}$ y que, además, son chunches que se pueden chinear. ${ }^{8}$ (Chaves, 2007)

A modo de conclusión, se debe de eliminar el concepto de "el patrimonio" porque perpetúa procesos y prácticas de "la colonialidad" en la población hetereogénea de Costa Rica. Además de recurrir a los procesos de "la descolonialidad", es importante emprender diálogos con los diversos sectores de la población heterogénea de Costa Rica con el fin de visibilizar y respetar sus fuentes de saberes/sistemas.

\section{BIBLIOGRAFÍA}

Autoridad Reguladora de Servicios Públicos. (s.f.). Resolución Alterna de Conflictos y Promoción de la Paz Social, Ley No 7727. En: Autoridad Reguladora de Servicios Públicos (ARESEP). Dirección: http://aresep.go.cr/images/documentos/Ley_resolucion_alternativa_c onflictos.pdf. Consulta: 9 de agosto de 2016.

Baltodano Román, G. \& Camacho Alfaro, M. (2010). Identidad, invensión y mito: ensayos escogidos. San José: Editorial de Costa Rica.

Briceño Porras, G. (2011, Octubre 19). Caracterización del discurso de Simón Rodríguez y su incidencia en la conceptualización del patrimonio cultural. Tesis para optar al título de Doctor en Patrimonio Cultural, Universidad Latinoamericana y del Caribe. Dirección: http://www.eumed.net/tesis-

doctorales/2012/gbp/bibliografia_uno.html. Consulta: 9 de agosto de 2016.

Butzonitch, M.M.A. (2009, Diciembre). Poder, patrimonio y democracia en Andamios, Revista de Investigación Social. Vol. 6 (Núm. 12) pp.11-40. En: Universidad Autónoma de la Ciudad de México. Dirección:

\footnotetext{
${ }^{5}$ corrongo: (adj.) gracioso, bonito.

${ }^{6}$ chuzo: (adj.) bueno, excelente, tuanis.

${ }^{7}$ chiva, chivísima: expresión entre los jóvenes que denota agrado. ¡Que chiva!

${ }^{8}$ chinear: (v.) dar cariño, mimar, tratar bien.
} 
http://www.latindex.ppl.unam.mx/index.php/search/searchResults. Consulta: 9 de agosto de 2016.

Campbell Barr, E. (s.f.). Afrodescendientes y Multiculturalismo según el Censo 2011. Costa Rica: Centro de Mujeres Afrocostarricenses. En: Instituto Nacional de Estadística y Censos (INEC). Dirección: http://www.inec.go.cr/sites/default/files/documentos/inec_institucion al/publicaciones/anpoblaccenso2011-03.pdf_0.pdf. Consulta: 9 de agosto de 2016.

Cañas, A. (2010). Uso y práctica del chunche. En G. Baltodano Román \& M. Camacho Alfaro, Identidad, invensión y mito: ensayos escogidos. San José: Editorial de Costa Rica.

Chaves, M. (2007, Marzo 4). Diccionario de costarriqueñismos. En: El callejón de los olvidos (Blog). Dirección: http://el-callejonm.blogspot.com/2007/03/diccionario-de-costarriqueismoslargo.html. Consulta: 9 de agosto de 2016.

De Sena, A. (2014). Notas sobre lo social como ámbito de debates no cerrados. En Paulo Henrique [et.al.] (Eds.), Guía sobre post-desarrollo y nuevos horizontes utópicos, pp.153-163. Argentina: Estudios Sociológicos Editora. Dirección: https://www.scribd.com/document/249873643/Guia-sobre-postdesarrollo-y-nuevos-horizontes-utopicos. Consulta: 9 de agosto de 2016.

Estado de la Nación. (2015). Seguimiento del desarrollo humano sostenible.Vigesimoprimer Informe Estado de la Nación. En: Estado de la Nación. Dirección: http://www.estadonacion.or.cr/21/assets/en-21cap-2.pdf. Consulta: 9 de agosto de 2016.

Fondo Indígena. (2007). Módulo de Historia y Cosmovisión Indígena. Guía de aprendizaje colectivo para organizaciones y comunidades. Bolivia: Escuela Intercultural de Gobierno y Políticas Públicas. Programa de Formación de Líderes Indígenas. En: Fondo Indígena. Dirección: 
http://www.fondoindigena.org/wpcontent/uploads/2011/08/Modulo-Historia.pdf. Consulta: 9 de agosto de 2016.

Fundación ILAM. (2016). Los diversos patrimonios. En: Fundación Instituto Latinoamericano de Museos y Parques. Dirección: http://www.ilam.org/index.php/es/programas/ilam-patrimonio/losdiversos-patrimonios?highlight $=W y J w Y X R y a W 1 v b m l v \| l 0=$. Consulta: 9 de agosto de 2016.

García Segura, A. (2003). Diccionario de mitología bribri. Costa Rica: Editorial Universidad de Costa Rica.

Grünberg, K. (2015). Cultural Heritage and Intellectual Property: Copyright Issues regarding the Digital Collections of Traditional Cultural Expressions (TCEs) and Traditional Knowledge (TK) of Indigenous Peoples in Museums, Libraries, Archives, and Cultural Institutions.

Dirección: de http://slanypublications.org/2015/01/18/culturalheritage-and-intellectual-property/. Consulta: 9 de agosto de 2016.

Guevara Berger, M. (ed.). (7 setiembre 2007). Perfil de los pueblos indígenas de Costa Rica. Informe Final. En: Cuasran. Dirección: http://cuasran.blogspot.com/2007/09/perfil-de-los-pueblosindgenas-de-costa.html. Consulta: 9 de agosto de 2016.

Henrich, I. F. (2014). Colonialidad del Saber, Pluralismo epistemológico y Modernidad. En P.H. Martins [et.al.] (Eds.), Guía sobre post-desarrollo y nuevos horizontes utópicos, pp. 41-49. Argentina: Estudios Sociológicos Editora. Dirección: https://www.scribd.com/document/249873643/Guia-sobre-postdesarrollo-y-nuevos-horizontes-utopicos. Consulta: 9 de agosto de 2016.

Instituto Interamericano de Derechos Humanos. (2016). El derecho a la consulta previa, libre e informada: una mirada crítica desde los pueblos indígenas. Costa Rica: Instituto Interamericano de Derechos Humanos. Dirección: http://www.iidh.ed.cr/IIDH/media/3703/el- 
derecho-a-la-consulta_una-mirada-cr\%c3\%adtica-desde-los-pueblosind\%c3\%adgenas.pdf. Consulta: 9 de agosto de 2016 .

Jaén García, L.F. (2006). El sistema nacional de información archivística como modelo de unificación de archivos. Costa Rica: Editorial Universidad de Costa Rica.

La Prensa Libre. (2015, Noveimbre 22). Costa Rica celebra diez años de la carreta y el boyero. La Prensa Libre. Direción: http://www.laprensalibre.cr/Noticias/detalle/48239/456/costa-ricacelebra-diez-anos-de-la-carreta-y-el-boyeo-. Consulta: 9 de agosto de 2016.

Mackay, F. \& Morales Garro, A. (2014). Violaciones de los derechos territoriales de los pueblos indígenas: el ejemplo de Costa Rica. Reino Unido: Forest Peoples Programme. En: Forest People. Dirección: http://www.forestpeoples.org/sites/fpp/files/publication/2014/02/viol ationsterritorialrightscostaricaenglishfeb2014.pdf. Consulta: 9 de agosto de 2016.

Mejía, J. (2008, Marzo). Epistemología de la investigación social en América Latina. Desarrollos en el Siglo XXI. Cinta Moebio, Vol. 31 pp.1-13. Dirección: http://www.scielo.cl/scielo.php?pid=S0717554X2008000100001\&script=sci_arttext. Consulta: 9 de agosto de 2016.

Ming, Y. (s.f.). Variación lingüística en Mamita Yunai. Universidad de Estudios Internacionales de Shanghái (SISU). En: Chulangkorn University. Dirección: http://www.arts.chula.ac.th/ west/spanish/wpcontent/uploads/sites/6/2016/01/Yang-Ming.pdf Consulta: 9 de agosto de 2016.

Ministerio de Cultura y Juventud de Costa Rica (s.f.a). Patrimonio cultural material.

Dirección: http://www.patrimonio.go.cr/patrimonio/material/index.aspx. Consulta: 9 de agosto de 2016. 
Ministerio de Cultura y Juventud de Costa Rica (s.f.b). Patrimonio cultural inmaterial.

Dirección:

http://www.patrimonio.go.cr/patrimonio/inmaterial/index.aspx.

Consulta: 9 de agosto de 2016.

Ministerio de Cultura y Juventud de Costa Rica. (2013). Política General de Derechos Culturales 2014-2023. Dirección: http://www.mcj.go.cr/ministerio/legislacion/02.pdf. Consulta: 9 de agosto de 2016.

Molina, L.E. (2007). La conservación del patrimonio cultural en Venezuela: Nuevas oportunidades a partir de 1999. Rev. Venez. de Econ. y Ciencias Sociales, Vol. 13 (Núm. 13) pp. 129-141. En: Universidad Central de Venezuela. Dirección: http://www.ucv.ve/fileadmin/user_upload/faces/problemas_sociales_c ontemporaneos/CESOC/SEPTIEMBRE_DICIEMBRE_3_2007_MIRADAS _ANTROPOLOGICAS_A_VENEZUELA.pdf. Consulta: 9 de agosto de 2016.

Municipalidad de Desamparados. (s.f.). Museo de la Carreta y el Campesino Costarricense.

Dirección: http://www.desamparados.go.cr/index.php/museo-de-lacarreta-y-el-campesino-costarricense. Consulta: 9 de agosto de 2016.

Museo Nacional de Costa Rica. (2016). Manual de normas y procedimientos para el tratamiento y el manejo de las colecciones arqueológicas con contexto. Costa Rica: Museo Nacional de Costa Rica.

Dirección: http://www.museocostarica.go.cr/descargas/Manual\%20Colecciones \%202016.pdf. Consulta: 9 de agosto de 2016.

Organización de las Naciones Unidas. (2008, Marzo). Declaración de las Naciones Unidas sobre los derechos de los pueblos indígenas. Dirección: http://www.un.org/esa/socdev/unpfii/documents/DRIPS_es.pdf. Consulta: 9 de agosto de 2016. 
Organización de las Naciones Unidas para la Educación, la Ciencia y la Cultura. (2005). Oxherding and Oxcart Tradition in Costa Rica. Dirección:

http://www.unesco.org/culture/intangible-heritage/12lac_uk.htm.

Consulta: 9 de agosto de 2016.

Organización de las Naciones Unidas para la Educación, la Ciencia y la Cultura. (2006). Obras Maestras del Patrimonio Oral e Inmaterial de la Humanidad Proclamaciones 2001, 2003 y 2005. Dirección: http://unesdoc.unesco.org/images/0014/001473/147344s.pdf.

Consulta: 9 de agosto de 2016.

Organización de las Naciones Unidas para la Educación, la Ciencia y la Cultura. (2015, Noviembre 17). Recomendación relativa a la protección y promoción de los museos y colecciones, su diversidad y su función en la sociedad. Dirección: http://portal.unesco.org/es/ev.phpURL_ID=49357\&URL_DO=DO_TOPIC\&URL_SECTION=201.html.

Consulta: 9 de agosto de 2016.

Palermo, Z. (2012). Colonialidad del poder y género: una historia local. Sociocriticism, Vol. 27 (Núm. 1-2) pp.15-43. En: Editorial Universidad de Granada.

Dirección: http://revistaseug.ugr.es/index.php/sociocriticism/article/view/2438/. Consulta: 9 de agosto de 2016.

Restrepo, E. \& Cabrera, M. (s.f.). Pensamiento decolonial: teoría crítica desde América Latina. En: Red de Antropologías del Mundo (RAMWAN). Dirección: http://ram-wan.net/restrepo/decolonial/. Consulta: 9 de agosto de 2016.

Walsh, C. (Marzo 2004). Geopolíticas del conocimiento, interculturalidad y descolonización, Boletín Instituto Científico de Culturas Indígenas-ARY Rimay, Vol. 6 (Núm. 60). En: Instituto Científico de Culturas Indígenas.

Dirección: http://icci.nativeweb.org/boletin/60/walsh.html. Consulta: 9 de agosto de 2016. 
Wikipedia. (2016a, May 1). Grupo modernidad/colonialidad. Dirección:

https://es.wikipedia.org/wiki/Grupo_modernidad/colonialidad.

Consulta: 9 de agosto de 2016.

Wikipedia. (2016b, Agosto 18). Patrimonio. Dirección: https://es.wikipedia.org/wiki/Patrimonio\#cite_note-2. Consulta: 9 de agosto de 2016.

Wikipedia. (2016c, Agosto 3). Símbolos patrios. Dirección: https://es.wikipedia.org/wiki/S\%C3\%ADmbolos_patrios_de_Costa_Ri ca. Consulta: 9 de agosto de 2016. 